\title{
Knowledge of General Practitioners in Cotonou about Systemic Lupus Erythematosus
}

\author{
Angèle Azon-Kouanou, Nonvignon Carrel Abdias Aboué, Mahoutin Semassa Ghislain Missiho, \\ Eugénie Dansou, Yves Morel Sokadjo, Kouessi Anthelme Agbodande, \\ Armand Finagnon Wanvoegbe, Djimon Marcel Zannou, Fabien Houngbé
}

Internal Medicine and Medical Oncology Department, National Teaching Hospital (NTH), Hubert Koudougou Maga (HKM), Cotonou, Bénin

Email: angele.azonkouanou@gmail.com

How to cite this paper: Azon-Kouanou, A., Aboué, N.C.A., Missiho, M.S.G., Dansou, E., Sokadjo, Y.M., Agbodande, K.A., Wanvoegbe, A.F., Zannou, D.M. and Houngbé, F. (2020) Knowledge of General Practitioners in Cotonou about Systemic Lupus Erythematosus. Open Journal of Internal Medicine, 10, 311-320.

https://doi.org/10.4236/ojim.2020.104032

Received: August 23, 2020

Accepted: October 23, 2020

Published: October 26, 2020

Copyright (c) 2020 by author(s) and Scientific Research Publishing Inc. This work is licensed under the Creative Commons Attribution International License (CC BY 4.0).

http://creativecommons.org/licenses/by/4.0/

\section{(cc) (i) Open Access}

\begin{abstract}
Introduction: Patients with Systemic Lupus Erythematosus (SLE) are seen late in specialized medical consultation in Benin. The objective of this work was to assess general practitioners' knowledge in Cotonou about SLE. Materials and Methods: This work was a cross-sectional study that was led in the city of Cotonou from July 1 to September 30, 2017. In the study population, we have general practitioners who practice in the city. Data collection was set in response to a self-questionnaire. Result: The survey involved 209 general practitioners. The average age was 27.5 years with a minimum of 22 and a maximum of 34 . Most of them practiced in private clinics. Besides, 17 doctors (8.1\%) reported that they never heard of lupus. Among the 192 remaining, only one had an average knowledge of lupus, while the others had insufficient knowledge. Conclusion: From this study, we got that SLE is still little known by the general practitioner. Increasing the knowledge of general practitioners' knowledge of lupus is compulsory to improve the screening rate.
\end{abstract}

\section{Keywords}

General Practitioners, Knowledge, Systemic Lupus Erythematosus

\section{Introduction}

Systemic Lupus Erythematosus is an auto-immune disease from the group of the connective tissue disease [1] [2]. In reality, it is a protean disease that becomes serious when it is not treated. It also affects women in periods of ovulatory activity (sex ratio 9 women of 1 man). Biologically, it is characterized by the production of antinuclear antibodies directed in particular against native DNA. It is sometimes associated with the anti-phospholipid antibody syndrome that is de- 
fined by the association of thrombosis or obstetrical events and anti-phospholipid antibodies. The vital prognosis depends on: the presence of some severe visceral disorders, mostly renal, the risk of infection, and cardiovascular complications [3].

In the Benin Republic, the published papers about this disease were done in numerous hospitals and patients are seen late by medical specialists, around 5 to 10 years on average after the onset of the first symptoms [4] [5]. This delay in diagnosis reveals a poor prognosis, since this long elapsed period before the implementation of a specific treatment leads to damage to vital organs and death. Several hypotheses have been put forward to explain this delay in diagnosis, including knowledge of first contact health professionals such as general practitioners. The low level of knowledge of these general practitioners on SLE could delay the referral of the patient to specialist doctors and consequently the initiation of a treatment that should slow the progression of the disease. This hypothesis supports the current work with the objective of assessing the level of knowledge of general practitioners about the diagnosis and management of lupus to establish a training program.

\section{Materials and Methods}

This work is a cross-sectional study conducted in the city of Cotonou from July 1 to September 30, 2017. In the study population, we have general practitioners and student doctors in their final school year who practice in the city. Let's note that, in our context, general practitioners are doctors. Additionally, student doctors are to become general practitioners. Actually, we did a complex two-stage exhaustive sampling. Firstly, we selected the health institutions (teaching hospitals, local hospitals and polyclinics, clinics and medical offices were represented), then the doctors practicing there who were selected and eligible were included in the study. In addition, data collection was done with a self-questionnaire assisted by the investigation agency. As we know, this study is about knowledge of a specific disease and considering the standard [6] about those kinds of questionnaires, we set our following the required process. Though its validity and reliability were already validated [6], we have also done a pilot study to check how likely the outputs were. We have in total 37 questions but they were structured into four sections. In other words, we have variables related to socio-demography, profession, knowledge-SLE, and practice-SLE. Every doctor's level of knowledge was labelled insufficient, average, fairly good, or good depending on whether they gave, respectively, less than $50 \%$, between 50 and $65 \%$, between 65 and $85 \%$ or more than $85 \%$, of correct answers [6]. The data sets were analyzed with CS Pro 7.0 and Excel 2016.

\section{Results}

\subsection{General Points}

Actually, we got 209 doctors in the study. There were 37 student doctors in their 
final school year in medicine and 172 general practitioners. Most of the doctors were from Benin medical faculty $(98.1 \%)$ and mainly from the Faculty of Health Sciences (FSS) in Cotonou (80.9\%). The average age was 27.5 years with a minimum of 22 and a maximum of 34 .

\subsection{Knowledge}

\subsubsection{Knowledge Score}

Most of the doctors (91.4\%) had limited knowledge about lupus. Doctors' knowledge scores are in Table 1.

The knowledge score has constantly increased from 2008 (17.9\%) to 2017 (31.1\%) regardless of the peak in 2009 as shown in Figure 1.

\section{- Information sources of Doctors who knew about lupus}

The main information source of SLE was the basic medical education (84.4\%). In addition, Table 2 describes the different types of doctors' information sources with some level of knowledge about lupus.

The main LES teaching during reported basic medical education was internal medicine (48.1\%) and dermatology (43.2\%).

\section{- Knowledge of clinical manifestations}

The knowledge of the existence of clinical manifestations related to LES is described in Table 3.

Table 1. Doctors' knowledge score about LES.

\begin{tabular}{cccc}
\hline Score & Knowledge & Frequency & Percentage (\%) \\
\hline $0 \%$ & Don't know & 17 & 8.1 \\
{$[0 \% ; 50 \%]$} & Limited & 191 & 91.4 \\
{$[50 \% ; 65 \%]$} & Average & 1 & 0.5 \\
{$[65 \% ; 85 \%]$} & Good enough & 0 & 0.0 \\
{$[85 \% ; 100 \%]$} & Good & 0 & 0.0 \\
Total & & 209 & 100.0 \\
\hline
\end{tabular}

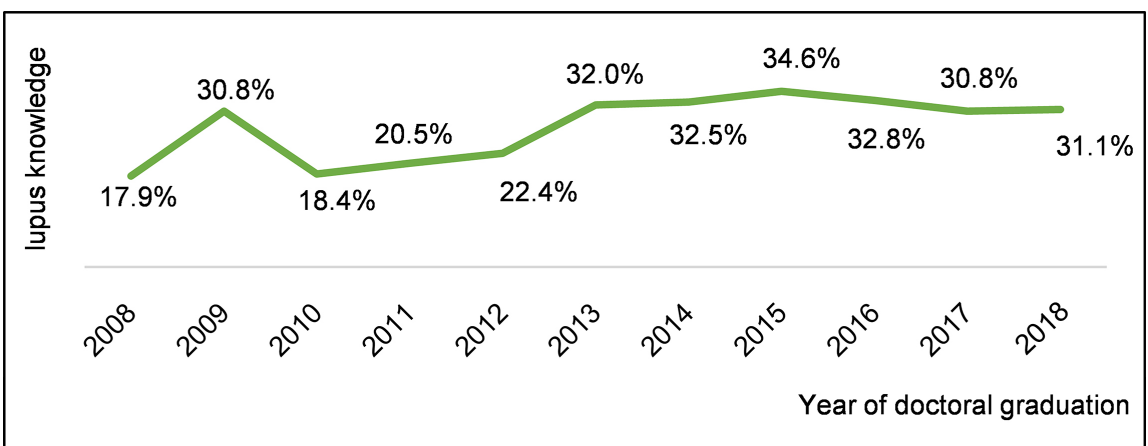

Figure 1. Evolution of knowledge score about SLE from 2008 to 2017. 
Cutaneous, general, and rheumatological manifestations were the most frequently mentioned by physicians. Namely, we have erythema vespertilio (63.5\%), fever (81.8\%), and arthralgia (62.0\%) that were, respectively, the most frequent symptoms of the manifestation.

\subsubsection{Contributing Factors}

In Table 4, we have the description of the factors that are said to contribute to the increase of SLE by doctors.

Pregnancy, sun exposure, and medication are the most common triggers that the interviewees listed.

Table 2. Distribution of general practitioner by SLE information source.

\begin{tabular}{ccc}
\hline & Frequency $(\mathrm{n}=192)$ & Percentage $(\%)$ \\
\hline Basic medical training & 162 & $\mathbf{8 4 . 4}$ \\
Mass Media & 24 & 12.5 \\
Medical Press & 21 & 10.9 \\
EPU & 15 & 7.8 \\
Others & 28 & 14.6 \\
\hline
\end{tabular}

${ }^{*}$ Others: LES personal or family history, conference, inter-university diploma.

Table 3. Knowledge of the existence of clinical manifestations related to LES among general practitioners.

\begin{tabular}{ccc}
\hline Known Manifestations & Frequency & Percentage \\
\hline Mucocusal skin manifestations & 173 & $90.1 \%$ \\
General manifestations & 171 & $89.1 \%$ \\
Rheumatologic manifestations & 167 & $87.0 \%$ \\
$\quad$ Renal manifestations & 159 & $82.8 \%$ \\
Hematologic manifestations & 157 & $81.8 \%$ \\
Cardiovascular manifestations & 151 & $78.6 \%$ \\
Neuropsychiatric manifestations & 146 & $76.0 \%$ \\
Breathing manifestations & 132 & $68.8 \%$ \\
Digestive manifestations & 121 & $63.0 \%$ \\
Ophthalmologic manifestations & 104 & $54.2 \%$ \\
Genital manifestations & 93 & $48.4 \%$ \\
Ear-nose-throat manifestations & 28 & $14.6 \%$
\end{tabular}


Table 4. Knowledge on the factors that are said to contribute to the increase of SLE by doctors.

\begin{tabular}{ccc}
\hline Known factors that can contribute to SLE & Frequency & Percentage \\
\hline Pregnancy & 133 & $69.3 \%$ \\
Exposition to sun & 117 & $60.9 \%$ \\
Drugs & 112 & $58.3 \%$ \\
Stress & 110 & $57.3 \%$ \\
Tobacco & 96 & $50.0 \%$ \\
Infections & 72 & $37.5 \%$ \\
Contact with allergen & 48 & $25.0 \%$ \\
Alcohol & 11 & $5.7 \%$ \\
Don't know & 8 & $4.2 \%$ \\
\hline
\end{tabular}

\subsubsection{Knowledge of General Practitioners about At-Risk Population} 14 doctors $(7.3 \%)$ declared that SLE has been regularly noticed with young black women. Respectively, $78.1 \%, 75.5 \%$, and $81.8 \%$ of doctors have confirmed that SLE is also possible with children, old people, and male patients. In addition, most of the surveyed (97.4\%) stated that SLE can be diagnosed to female people.

\subsubsection{Very Complicated Manifestations}

Among the clinical manifestations of SLE, those that are cardiovascular and neuropsychiatric are the most frequently listed to be complicated. The ENT manifestations are the least mentioned in that section.

\subsubsection{Support of Immunology to the Diagnostic}

The majority of doctors (71.3\%) said that they did not know the antibodies in SLE. Among the 192 practitioners who heard of LES, 43 (20.6\%) cited native anti-DNA antibodies and 27 (12.9\%) cited native anti-DNA antibodies as being specific for SLE.

\section{- Criteria of classification}

The majority of physicians (154\% or $80.2 \%$ ) reckoned that they did not know the classification criteria for LES. Among the 38 remaining, 17.2\% mentioned the ACR classification and $1.6 \%$ the SLICC.

\section{- Associated pathologies}

Gougerot-Sjögren syndrome is the pathology frequently associated with SLE that is most cited by physicians (44.5\%), followed by anti-phospholipid syndrome (18.2\%). We also have 66 doctors (31.6\%) who did not know any of the pathologies frequently associated with SLE.

\section{- Therapeutic modality}

\subsubsection{Used Molecules}

Among the 192 physicians, 10 (5.2\%) noted both corticosteroids and synthetic 
antimalarials as part of the initial background therapy.

\subsubsection{Duration}

Most of the doctors (153\% or $79.3 \%$ ) declared that they did not know the duration of SLE treatment.

\section{- Training request}

Most of the surveyed doctors who have heard of SLE (189\% or $98.4 \%$ ) expressed the desire to participate in a training on this disease.

\section{Discussion}

\subsection{General Points}

Our study population was general practitioners and final school year students. Their age on average was 27.5 years and their average seniority period was 2.9 years. This population is quite young compared to the one in the studies of Rat et al. concerning the management of rheumatoid arthritis in general practice where the average age was 49 years [7] or the work of Badoum et al. on the knowledge, attitudes and practices of general practitioners on asthma in Ouagadougou, where the average age was 34 years [8]. Most of the doctors were graduated from the faculties of Benin. Our study was indeed led in Cotonou where the largest and oldest medical faculty in the country is located. Thus, doctors who recently got graduated or are waiting to defend, work in that city of their training faculty or in neighboring localities while waiting for more stable professional projects. This situation also explains the relatively low average age and seniority length.

Most of the doctors worked in polyclinics or local hospitals. Actually, these centers rarely offer permanent contracts. Indeed, these health facilities tend to employ a greater number of general practitioners and students to ensure continuity and on-call duty to manage the flow of patients.

Clinics and medical offices generally employ a single doctor who is in charge of ensuring continuity and not on-call.

Teaching hospitals most often offer stable jobs. However, these are the reference teaching hospitals where most of the practicing doctors are specialists or are in the process of being specialists.

\subsection{Knowledge of Doctors about SLE}

The current results of the study revealed a low level of knowledge of general practitioners about SLE. Actually, 17 doctors (8.1\%) stated not to have heard about this disease. Among the 192 physicians remaining, 191 had a limited knowledge of SLE. Blaauw et al. in 1995 demonstrated that it was one of the most difficult diagnoses for general practitioners [9]. This finding in our work has also been noticed in the case of several other diseases in different other studies. Thus, Rat et al. in their studies related to general practitioners in the management of rheumatoid arthritis confirmed that there are important gaps in the 
knowledge of general practitioners on this disease [7] [8]. The analyses of these studies allow us to identify the lack in the care of patients, due to a lack of theoretical knowledge of general practitioners, in fact in numerous majors of medicine. Bloy [10] also noted a notion of uncertainty that is attached to the condition of general practitioners. According to her, this uncertainty is due to the relationship of the general practitioner to medical knowledge and social relations that are required by his position. In the first case, the uncertainty is deployed on three different levels: a level 1 that "results from an incomplete or imperfect mastery of the available knowledge", a level 2 that "depends on the limits related to medical knowledge" these days, and a level 3 that is due to the issue for a given practitioner to balance for the first and second levels. She even wrote that among all the doctors, the general practitioner is the one who deals with the most extensive field of knowledge, even infinitely, his "specialty" is not well limited a priori. "Incomplete or imperfect mastery of available knowledge" is therefore its concern, and even more.

Whatever personal efforts he makes or the quality of his initial training, the general practitioner has become, and will remain, the one who knows a little of everything.

In our study, we find that the primary source of information for surveyed doctors about SLE was their initial medical education. Internal medicine was the main teaching discipline which was followed by the dermatology. In the content of the doctors training at the Cotonou FSS, lupus has been taught in the dermatology program for several decades and in internal medicine for a year. This shows the need to strengthen the knowledge of general practitioners through postgraduate teaching. The level of knowledge, and therefore the level of uncertainty, changes with the addressed points in the different majors of medical knowledge. Thus, the factor contributing the most and cited by doctors was pregnancy (69.3\% or 133), and it is followed by exposure to the sun and some drugs. This is because they are the most frequently found factors [11].

The best known manifestations are general, mucosal skin, and rheumatic. They are also the most frequent and common manifestations of systemic lupus [12] [13].

Among the general manifestations, fever was the most cited; it is also the most common general sign; however it is not always related to SLE itself but rather to infectious complications of the treatment [14].

The most well-known mucosal skin manifestation by physicians was erythema malaria vespertilio. It is one of the specific lesions of SLE and it gives the disease its etymological origin [14].

Only $44.8 \%$ of physicians who reported the existence of mucosal skin manifestations in SLE mentioned discoid lesions, among them, 27.6\% cited skin necrosis, and a quarter of them chose oral erosions. Urticarial lesions, alopecia, livedo, and vasculitis lesions are the least mucosal skin manifestations that were revealed. This is somehow an evidence of the fact that despite the great specificity 
of these different lesions [14], they are still not well understood by general practitioners. Among the rheumatic manifestations, the most cited by doctors were arthritis and arthralgia (62.0\%) and myalgia (49.0\%). These manifestations are indeed among the most frequent of the group of rheumatologic manifestations [14]. Jaccoud's deformation was the least mentioned despite its great specificity [14].

Among physicians, $82.8 \%$ are aware of the existence of renal manifestations of SLE, but only $21.5 \%$ know about the prognostic importance of these attacks. The determining nature of these manifestations in the prognosis [12] explains their systematic research by specialist practitioners and it could explain the fact that general practitioners know their existence but not their characteristics, including prognostic importance. Thus, the almost constant glomerular character of renal involvement, for example [14], was not known to a third of the doctors.

The cardiovascular manifestations of SLE have been enumerated [14], among the most serious by physicians. However, they are not well known to doctors since the most frequently mentioned endocarditis has only been mentioned twice in five.

The neuropsychiatric manifestations of SLE have also been correctly cited among the most serious. They are also poorly understood since hallucinations, delusions, psychosis, and confusional states, although not frequent and not very specific [14], were the most cited. However, strokes and seizures, found frequently in patients, were mentioned by less than a third of physicians.

The best-known breathing manifestation of SLE by physicians was pleurisy. It was cited by $41.1 \%$ of them. It is also one of the most common breathing manifestations [14]. The most mentioned hematologic manifestation by physicians was the acceleration of SV. Hemolytic anemia was only cited by $28.1 \%$ of physicians despite its high frequency [14].

Only $12.9 \%$ of physicians cited native anti-DNA antibodies among the antibodies to be assayed in SLE. However, these are the most specific antibodies to the disease [12] [14]. Regarding the classification criteria, those of the American College of Rheumatology (ACR) were chosen by $17.2 \%$ of physicians, those of the SLICC by only $1.6 \%$. Trépos et al., in their study of general practitioners and medical recommendations have shown that while they approve of the growing role of recommendations in medical practice and strive to take it into account, general practitioners rely primarily on their experience of practitioners to sort through the mass of recommendations they received. The knowledge of general practitioners on the diagnosis of lupus is thus limited.

\section{Conclusion}

General practitioners are relatively young. Their level of knowledge related to lupus disease is generally limited, though the main source is basic medical training. Consequently, it is important to continuously strengthen the capacities of physicians in the area of lupus disease both in postgraduate studies and during 
initial basic training.

\section{Study Limitation}

The current work has a main limitation and it is compulsory to output it. Let's emphasize that all the medical agents that we questioned schooled in the same university and this might influence the variability in the answers about knowledge of SEL.

\section{Authors' Contributions}

All authors contributed to the conduct of this work and to the writing of the manuscript.

\section{Conflicts of Interest}

The authors declare no conflicts of interest regarding the publication of this paper.

\section{References}

[1] Feldman, C.H., Hiraki, L.T., Liu, J., Fischer, M.A., Solomon, D.H., Alarcón, G.S., Winkelmayer, W.C. and Costenbader, K.H. (2013) Epidemiology and Sociodemographics of Systemic Lupus Erythematosus and Lupus Nephritis among US Adults with Medicaid Coverage, 2000-2004. Arthritis \& Rheumatology, 65, 753-763.

https://pubmed.ncbi.nlm.nih.gov/23203603/

https://doi.org/10.1002/art.37795

[2] Saraux, A., Jousse, S., Roudaut, A. and Devauchelle, V. (2005) Epidemiology of Systemic Lupus Erythematosus. Rheumatism Review, 72, 117-119. https://doi.org/10.1016/j.rhum.2004.11.006

[3] High Authority of Health (2020) National Protocol of Care and Diagnostic: Systemic Lupus. HAS, Paris. http://www.has-sante.fr

[4] Petri, M. (2002) Epidemiology of Systemic Lupus Erythematosus. Best Practice \& Research: Clinical Rheumatology, 16, 847-858. http://www.ncbi.nlm.nih.gov/pubmed/12473278 https://doi.org/10.1053/berh.2002.0259

[5] Zomalheto, Z., Assogba, M., Agbodande. A., Atadokpede, F., Gounongbe, M. and Avimadje, M. (2014) Systemic Lupus Erythematosus: Particularities in Benin and West Africa. La Tunisie Médicale, 92, 707-710.

[6] Essi, M.J. and Oudou, N. (2013) The CAP Survey (Knowledge, Attitudes, Practices) in Medical Research. Health Sciences and Disease, 14, 1-3.

[7] Rat, A.C., Henegariu, V. and Boissier, M.C. (2006) The Prevalence of Patients Covered $100 \%$ by the French General Scheme. Revue du Rhumatisme, 73, 256-262. https://doi.org/10.1016/j.rhum.2005.02.005

[8] Badoum, G., Ouédraogo, S., Lankoandé, H., Ouédraogo, G., Boncoungou, K., Bambara, M. and Ouédraogo, M. (2012) Knowledge, Attitudes and Practices of General Practitioners on Asthma in Ouagadougou. Le Mali Medical, 27, 45-48.

[9] Rat, A.C., Henegariu, V. and Boissier, M.C. (2004) The Role of the General Practitioner in the Management of Rheumatoid Arthritis. Revue du Rhumatisme, 71, 350-358. https://doi.org/10.1016/j.rhum.2003.09.012 
[10] Bloy G. (2008) Uncertainty in General Medicine: Sources, Forms and Possible Accommodations. Sciences Sociales et Sante, 26, 67-91. https://doi.org/10.3406/sosan.2008.1881

[11] Bonnetblanc, J.M. (2012) Disseminated Lupus Erythematosus. Antiphospholipid Syndrome. Annales de Dermatologie et de Vénéréologie, 139, 102-111. https://doi.org/10.1016/j.annder.2012.06.006

[12] Arnaud, L., Mathian, A. and Amoura, Z. (2011) Clinical and Biological Manifestations of Systemic Lupus Erythematosus. La Revue du Praticien, 61, 1254-1258.

[13] Meyer, O. (2005) Systemic Lupus Erythematosus. EMC-Rhumatology-Orthopedics, 2, 1-32. https://doi.org/10.1016/j.emcrho.2004.08.005

[14] Meyer, O. (2013) Clinical and Biological Manifestations. Diagnosis of Lupus Erythematosus. Systemic Lupus, 41-72.

https://doi.org/10.1016/B978-2-294-71447-4.50009-X 